\title{
Characterization of a Tryptophan 2-Monooxygenase Gene from Puccinia graminis f. sp. tritici Involved in Auxin Biosynthesis and Rust Pathogenicity
}

\author{
Chuntao Yin,, Jeong-Jin Park, ${ }^{2}$ David R. Gang, ${ }^{2}$ and Scot H. Hulbert ${ }^{1}$ \\ ${ }^{1}$ Department of Plant Pathology and ${ }^{2}$ Institute of Biological Chemistry, Washington State University, Pullman 99164-6430, \\ U.S.A.
}

Submitted 7 October 2013. Accepted 11 December 2013.

\begin{abstract}
The plant hormone indole-3-acetic acid (IAA) is best known as a regulator of plant growth and development but its production can also affect plant-microbe interactions. Microorganisms, including numerous plant-associated bacteria and several fungi, are also capable of producing IAA. The stem rust fungus Puccinia graminis f. sp. tritici induced wheat plants to accumulate auxin in infected leaf tissue. A gene (Pgt-IaaM) encoding a putative tryptophan 2-monooxygenase, which makes the auxin precursor indole-3acetamide (IAM), was identified in the $P$. graminis $\mathbf{f}$. sp. tritici genome and found to be expressed in haustoria cells in infected plant tissue. Transient silencing of the gene in infected wheat plants indicated that it was required for full pathogenicity. Expression of Pgt-IaaM in Arabidopsis caused a typical auxin expression phenotype and promoted susceptibility to the bacterial pathogen Pseudomonas syringae pv. tomato $\mathrm{DC} 3000$.
\end{abstract}

Hormones regulate many aspects of plant growth, development, and appropriate responses to environmental stress, including phytopathogenic microbes. Salicylic acid (SA), jasmonic acid, and ethylene have well-documented roles in regulating defense responses (Bari and Jones 2009; Glazebrook 2005; Grant and Lamb 2006; Robert-Seilaniantz et al. 2011). In recent years, other hormones, especially auxin, have also been reported to associate with plant defense and microbial pathogenesis (Kazan and Manners 2009). For example, antibacterial resistance is enhanced by repressing auxin signaling in Arabidopsis (Navarro et al. 2006). The Pseudomonas syringae effector AvrRpt2 modulates auxin biosynthesis and promotes auxin signaling by stimulating the turnover of negative regulators in Arabidopsis and, therefore, promotes disease (Chen et al. 2007; Cui et al. 2013). Wang and associates (2007) demonstrated that the inhibition of auxin responses is a part of the SA-mediated disease-resistance mechanism in Arabidopsis. Other studies have indicated that auxin promotes susceptibility to $P$. syringae independently from SA signaling (Chen et al. 2004; Mutka et al. 2013). The discrepancies between these studies may be caused by different experimental conditions. Wang and associates (2007) pretreated plants exogenously with high concentrations of hormones, which may

Corresponding author: S. H. Hulbert; Telephone: +1.509.335.3722; Fax: +1.509.335.9581; E-mail: scot_hulbert@wsu.edu

(C) 2014 The American Phytopathological Society have different effects than if auxin levels are elevated as a result of increased biosynthesis of the hormone in plant cells or specific tissues (Mutka et al. 2013). Collectively, these findings indicate that reductions of auxin levels enhance plant defense responses.

Microorganisms are also capable of producing indole-3-acetic acid (IAA). Tryptophan has been identified as a main precursor for IAA biosynthesis pathways in bacteria. The indole-3-acetamide (IAM) pathway is the best characterized but not the only pathway and includes two distinct steps. Tryptophan is first converted to IAM by the enzyme tryptophan-2-monooxygenase (IaaM) (encoded by the IaaM gene) and then IAM is converted to IAA by an IAM hydrolase (IaaH) (encoded by the $\mathrm{IaaH}$ gene). The IAM pathway is mainly found in plant-associated bacteria, such as Agrobacterium tumefaciens, $P$. syringae, P. savastanoi, and Pantoea agglomerans (Patten and Glick 1996; Spaepen and Vanderleyden 2011). A few fungi, such as Colletotrichum gloeosporioides and Fusarium proliferatum (Maor et al. 2004; Robinson et al. 1998; Tsavkelova et al. 2012), also produce IAA via the IAM pathway. The IAA produced by bacterial pathogens is important for pathogenesis (Comai and Kosuge 1982; Spaepen and Vanderleyden 2011; Surico et al. 1985). Pathogenic strains of Erwinia herbicola were found to use the IAM pathway for the production of IAA, whereas nonpathogenic strains were devoid of this pathway (Manulis et al. 1991). Deactivating the IAM pathway by disrupting either the IaaM or IaaH genes reduced the virulence of E. herbicola pv. gypsohhilae on Gypsophila paniculata (Clark et al. 1993). IAA has been also demonstrated to promote gall formation. An IAA-deficient mutant of Pseudomonas savastanoi did not produce galls on host plants (Smidt and Kosuge 1978; Surico et al. 1985) but the ability to produce galls was restored when the mutant was transformed with genes for IAA synthesis (Comai and Kosuge 1982). In contrast, multiple mutants of Ustilago maydis greatly reduced IAA levels but were still pathogenic and caused gall formation on maize similar to wild-type strains (Reineke et al. 2008). Other studies have demonstrated that IAA has functions in fungi that are independent of interactions with plants. For example, IAA reduced the "spore density effect" on germination in Neurospora crassa (Nakamura et al. 1982). Exogenous IAA induced pseudohyphal growth in Saccharomyces cerevisiae (Prusty et al. 2004) and hyphal growth in Candida albicans (Rao et al. 2010).

In order to facilitate infection, plant pathogens deliver numerous effector proteins into the plant cells to promote their survival and growth in the host environment by altering hostcell structure and function (De Wit et al. 2009; Koeck et al. 
2011; Schmidt and Panstruga 2011). Proteins from biotrophic fungi and oomycetes that are secreted from haustorial cells are potential protein effectors and sometimes interact with resistance gene proteins. For instance, four avirulence (Avr) genes identified from the flax rust fungus Melampsora lini (AvrL567, AvrM, AvrP4, and AvrP123) encoded small secreted proteins expressed in haustoria (Catanzariti et al. 2006; Dodds et al. 2004). Similarly, a number of $A v r$ genes (Avrb-1, Avr3a, ATR13, ATR1NdWsB, Avrla, Avr4/6, Avr4, and AvrBlb1) from oomycete pathogens have been identified that also encode small secreted proteins (Birch et al. 2009; Morgan and Kamoun 2007). Two Puccinia graminis f. sp. tritici effector proteins that interact with the RPG1 resistance protein of barley were not predicted to be secreted by sequence analysis methods but were present on spore surfaces (Nirmala et al. 2011). Avr genes (Avr-alO and Avr-kl) with no apparent secretion signals have also been characterized from the barley powdery mildew fungus, Blumeria graminis pv. hordei, but it is not known if they are secreted from haustoria (Ridout et al. 2006).

Whereas protein effectors from biotrophic fungi have received considerable attention, small molecule effectors such as hormones have not. Haustorial expression of biosynthetic enzymes for metabolites with biological activity in plant cells may be an indication of small molecule effectors in these fungi. The purpose of this study was to characterize a $P$. graminis f. sp. tritici gene encoding a putative tryptophan 2-monooxygenase, which is highly expressed in rust haustoria cells and potentially involved in auxin biosynthesis and rust pathogenicity.
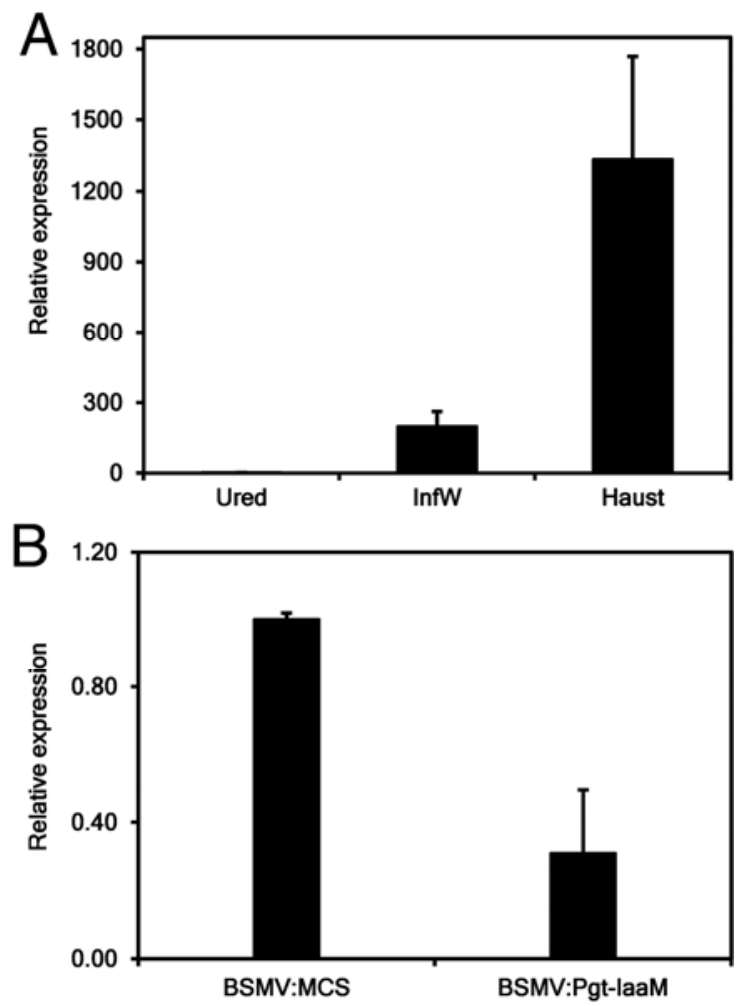

Fig. 1. Reverse-transcriptase quantitative polymerase chain reaction-based assessment of Pgt-IaaM gene expression. A, Pgt-IaaM gene expression in different developmental stages in rust. Ured: urediniospores; InfW: infected wheat leaves; and Haust: purified haustoria. B, Pgt-IaaM gene expression after silencing by BSMV-HIGS compared with the BSMV: MCS control. BSMV:MCS: plants infected with BSMV control and Pgt7A; BSMV:Pgt-IaaM: plants infected with the BSMV:Pgt-IaaM and Pgt7A. All data were normalized against the actin gene of Puccinia graminis f. sp. tritici. Standard deviation was calculated from values obtained from three biological replicates.

\section{RESULTS}

\section{A $P$. graminis gene encodes}

a putative tryptophan 2-monooxygenase.

Auxins have been observed to increase in rust-infected wheat tissues, although the control of this increase is not known (Artemenko et al. 1980; Bushnell 1984). A P. graminis f. sp. tritici gene, PGTG_11658 (herein Pgt-IaaM), encoding a predicted 588-amino-acid tryptophan 2-monooxygenase, was found in the Broad Institute Puccinia database. It had $82 \%$ amino acid identity with a predicted protein (PTTG_06071) from $P$. triticina and $79 \%$ identity with a predicted protein (CQM-04507) in P. striiformis. In contrast, no homologous Melampsora sequences were identified. Homologous genes were identified in several non-rust fungi, including several Fusarium spp., Glomerella graminicola, Colletotrichum gloeosporioides, and Neofusicoccum parvum (Tsavkelova et al. 2012). The genes in the three Puccinia spp. were found on sequence contigs carrying several common genes, indicating that they are on partially syntenic chromosome regions of at least 60 to $100 \mathrm{~kb}$. The partially syntenic region included genes encoding a mannose-1-phosphate guanyltransferase, a serine/threonine protein kinase, and several hypothetical proteins. In bacteria and fungi where the IAM pathway has been characterized, the genes encoding the two catalytic enzymes are often adjacent to each other in the genomes (Robinson et al. 1998; Tsavkelova et al. 2012). However, IaaM homologs were identified in the three Puccinia spp., while IaaH homologs were not present on the chromosomes near these genes or anywhere else in the assembled genomes.

\section{Regulation of Pgt-IaaM expression during rust infection.}

Pgt-IaaM mRNA expression during rust development in plants was examined by reverse-transcriptase quantitative polymerase chain reaction (RT-qPCR) analysis. Relatively low transcript levels were detected in urediniospores of $P$. graminis. Transcript levels in fungal cells growing in infected leaves were estimated to be almost 200 times higher than in urediniospores, suggesting that Pgt-IaaM expression is induced in the biotrophic growth phase (Fig. 1A). When transcript levels in purified haustorial cells were compared with total fungal cells in infected leaves, they were estimated to be approximately seven times higher in the haustorial cell preparations. Although infected leaves contain haustoria in addition to other cell types, the higher levels in the haustorial preparations indicate that the transcripts are much more highly expressed in haustoria. Therefore, the transcripts appear to be haustoria specific or highly enriched in haustorial cells. No amino terminal sequences were identified using the SignalP 4.1 or iPSORT to indicate that the protein was secreted from the haustorial cells.

\section{Hormone levels in urediniospores and $P$. graminis infected plants.}

Hormone levels were measured in different rust-infection stages and rust urediniospores. Rust urediniospores harvested from greenhouse-grown plants contained free IAA at $1.46 \pm$ $0.55 \mathrm{ng} / \mathrm{g}$ fresh weight $(\mathrm{FW})$. When auxin levels were measured in infected wheat leaves, very little IAA $(0.01 \pm 0.01 \mathrm{ng} / \mathrm{g}$ FW) was detected at 2 days after stem rust infection. IAA increased to $3.28 \pm 0.09 \mathrm{ng} / \mathrm{g} \mathrm{FW}$ at 4 days postinfection (dpi), with higher levels $(4.92 \pm 0.66 \mathrm{ng} / \mathrm{g} \mathrm{FW})$ at $6 \mathrm{dpi}$. Only trace amounts of IAA ( 0.01 to $0.02 \mathrm{ng} / \mathrm{g} \mathrm{FW}$ ) were detected in healthy leaves of control plants (Fig. 2A). The levels of IAM were analyzed and no significant difference was observed in healthy and rust-infected wheat leaves over the same time course (data not shown). 
Levels of abscisic acid (ABA) and trans-zeatin (tZ) were also measured for comparison. Urediniospores carried extremely low levels (Fig. 2B and C) of both hormones, possibly due to contamination from the host tissues. ABA levels increased in
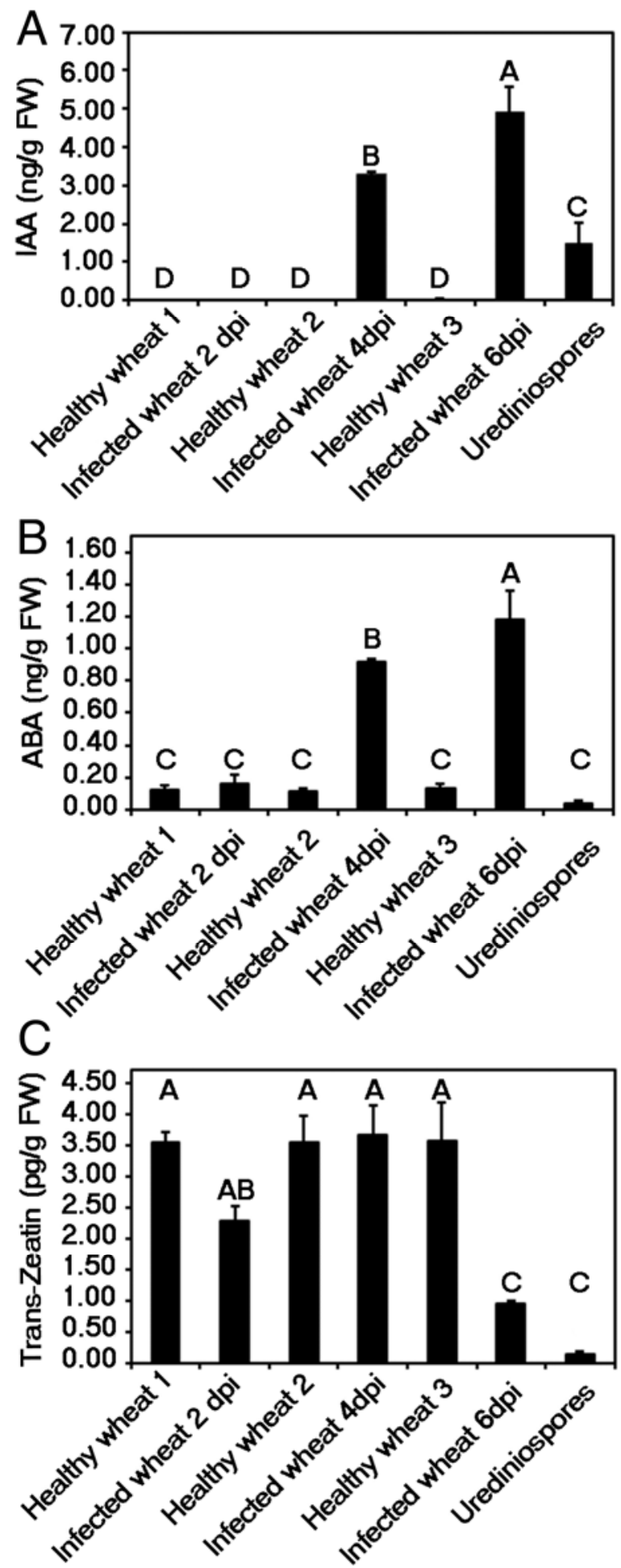

Fig. 2. Hormone levels in different rust-infected stages in wheat and rust urediniospores. A, Free indole-3-acetic acid (IAA) levels; B, abscisic acid (ABA) levels; $\mathbf{C}$, trans-zeatin levels. Healthy wheat 1 is uninfected wheat at the same stage as infected wheat at 2 days postinfection (dpi); Healthy wheat 2 is uninfected wheat at the same stage as infected wheat at $4 \mathrm{dpi}$; Healthy wheat 3 is uninfected wheat at the same stage as infected wheat at 6 dpi. Values are means and standard errors of three replicates. Different letters above columns indicate significant differences for hormone levels ( $P \leq 0.05$; Turkey's honestly significant difference test with $\alpha=0.05)$. infected plants over a time course similar to that of IAA; no noticeable increase at 2 dpi but increasing through 4 and 6 dpi. Patterns of $\mathrm{tZ}$ accumulation were very different, with levels similar to the uninoculated control plants at 2 and 4 dpi but reduced levels at $6 \mathrm{dpi}$. Overall, the results show that, by $6 \mathrm{dpi}$, when the rust fungus is well spread through the host tissue, the leaves contain elevated levels of IAA and ABA compared with healthy plants and reduced levels of $\mathrm{tZ}$.

\section{Silencing of Pgt-IaaM by host-induced gene silencing reduces the pathogenicity of $\boldsymbol{P}$. graminis.}

To investigate the function of Pgt-IaaM in the wheat-stem rust interaction, silencing was conducted using Barley stripe mosaic virus (BSMV)-mediated host-induced gene silencing (HIGS). First and second leaves of 12-day-old 'McNair 701' wheat were rub inoculated with the transcripts from either the BSMV construct that carried an RNA interference target region of Pgt-IaaM or the control virus consisting of the same vector without the fungal DNA fragment incorporated into the multiple cloning site of the $\gamma$ genome. After 10 days, those leaves displaying mild virus symptoms (pale yellow stripes on the leaves) were inoculated with $P$. graminis isolate Pgt7A. Samples of the infected leaves were harvested at $5 \mathrm{dpi}$ for RT-qPCR analysis to determine the extent of gene PgtIaaM silencing. The remaining infected plants were kept in the growth chamber and the infection types (IT) were scored at 12 dpi. The fungal disease phenotype displayed a reduction in the size of uredinia compared with BSMV:MCS controls (Fig. 3), where the only addition to the viral genome is a multiple cloning site from pBluescript K/S. Using the 0-to-4 scale described by Stakman and associates (1962), the plants inoculated with the BSMV:Pgt-IaaM construct often exhibited moderately resistant (MR) phenotypes, with rust IT of 2 to $2+$, but the control plants inoculated with BSMV:MCS or no BSMV consistently exhibited susceptible IT of 4 . Although some plants inoculated with the BSMV:Pgt-IaaM construct showed fully susceptible interactions, others showed the MR phenotype in every experiment whereas the control plants never did, and the average reaction type was signifi-

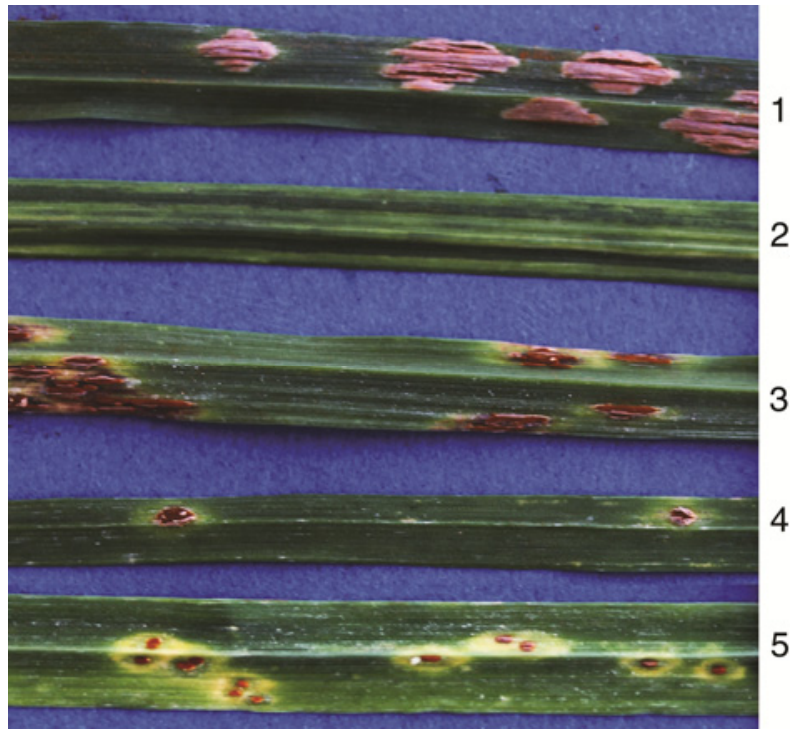

Fig. 3. Reduced virulence of Pgt7A on 'McNair 701' wheat after infection with BSMV:Pgt-IaaM. Row 1, McNair 701 challenged with Pgt7A; row 2, McNair 701 challenged with BSMV:MCS control; row 3, McNair 701 challenged with Pgt7A and BSMV:MCS control; row 4, McNair 701 challenged with Pgt7A and BSMV:Pgt-IaaM; row 5, 'Sr31/6*LMPG' wheat carrying the $\mathrm{Sr} 31$ gene challenged with Pgt7A. Picture was taken at 12 days postinoculation with Pgt7A. 
cantly lower $(P<0.05)$ in the BSMV:Pgt-IaaM-infected plants than the control plants. The effect of the small interfering RNA molecules on the Pgt-IaaM transcript levels was examined by RT-qPCR assays. Significant reduction in Pgt-IaaM transcript abundance was detected in infected wheat leaves at $5 \mathrm{dpi}$ and the average levels of Pgt-IaaM expression in silenced plants were approximately $31 \%$ of the control plants (Fig. 1B). These results indicate that Pgt-IaaM is required for full pathogenicity of $P$. graminis.

\section{Expression of Pgt-IaaM in Arabidopsis displays pleiotropic auxin-related phenotypes.}

Pgt-IaaM was predicted to code for tryptophan 2-monooxygenase, an enzyme that catalyzes the first step in the IAM pathway to synthesize auxin from tryptophan. To determine whether it is functional in auxin biosynthesis and whether expression of a single protein would increase auxin production in a plant, the gene was expressed in Arabidopsis thaliana biotype Columbia-0 (Col-0). The full predicted coding region was amplified from cDNA and inserted into a binary vector, pCHF3, that controls transcription with a Cauliflower mosaic virus $35 \mathrm{~S}$ promoter. Three independent transgenic Arabidopsis lines that expressed the gene were generated by Agrobacterium-mediated transformation. All three 35S:Pgt-IaaM transgenic lines displayed pleiotropic auxin-related phenotypes (Fig. 4). The 5-day-old transgenic seedlings exhibited approximately threefold longer hypocotyls and 1.5-fold longer primary roots than wild-type Col-0 (Fig. 4A and B). Four-weekold transgenic plants displayed narrow and downward-curling leaves (Fig. 4C). The transgenic plants also exhibited strong apical dominance and the height of fully grown transgenic plants was approximately twice that of wild-type plants (Fig. 4D). In addition, adult transgenic plants had twisted inflorescence stems and reduced seed set in many siliques (data not shown). RT-PCR analysis confirmed that phenotypes observed in 35S:Pgt-IaaM transgenic lines resulted from the accumula- tion of Pgt-IaaM transcript (Fig. 4E). IAA and IAM levels were measured in wild-type plants and 35S:Pgt-IaaM transgenic plants at different developmental stages and tissues. The free IAA levels in transgenic plants were higher than wild-type plants in all the tissues tested. Ten-day-old seedlings, 4-weekold rosette leaves, 6-week-old cauline leaves, and flowers of transgenic plants contained approximately six, eight, three, and two times more free IAA than the wild type, respectively (Table 1). Similar to that of IAA, IAM levels in transgenic plants were much higher than wild-type plants in all the tissues tested. Four-week-old rosette leaves, 6-week-old cauline leaves, and flowers of transgenic plants contained approximately $20,14,3$, and 36 times more IAM than the wild type, respectively. The IAM level of 10-day-old seedlings in nontransgenics was undetectable but trace amounts were detected in the other tissues tested (Table 1). These results indicate that the Pgt-IaaM gene functions in auxin synthesis.

\section{Expression of Pgt-IaaM in Arabidopsis promotes susceptibility to the bacterial pathogen Pseudomonas syringae pv. tomato DC3000.}

To further investigate the function of the Pgt-IaaM gene in pathogenesis, 4-week-old 35S:Pgt-IaaM transgenic Arabidopsis and Col-0 untransformed plants were challenged with a low level of Pseudomonas syringae pv. tomato DC3000 inoculum $\left(10^{4} \mathrm{CFU} / \mathrm{ml}\right)$. At $4 \mathrm{dpi}$, wild-type Col-0 leaves developed chlorotic lesions with mild necrosis, whereas DC3000 inoculation caused more severe yellowing and necrosis in leaves of all three transgenic lines (Fig. 5A). The growth of DC3000 inside the inoculated leaves was also measured. DC3000 multiplied 10 times more inside the inoculated leaves of transgenic lines than the Col-0 plants (Fig. 5B). Thus, the elevated growth of DC3000 in the transgenic lines was consistent with the observed disease phenotypes. Together, these data demonstrate that expression of Pgt-IaaM in Arabidopsis enhanced virulence of DC3000 growing on Arabidopsis.
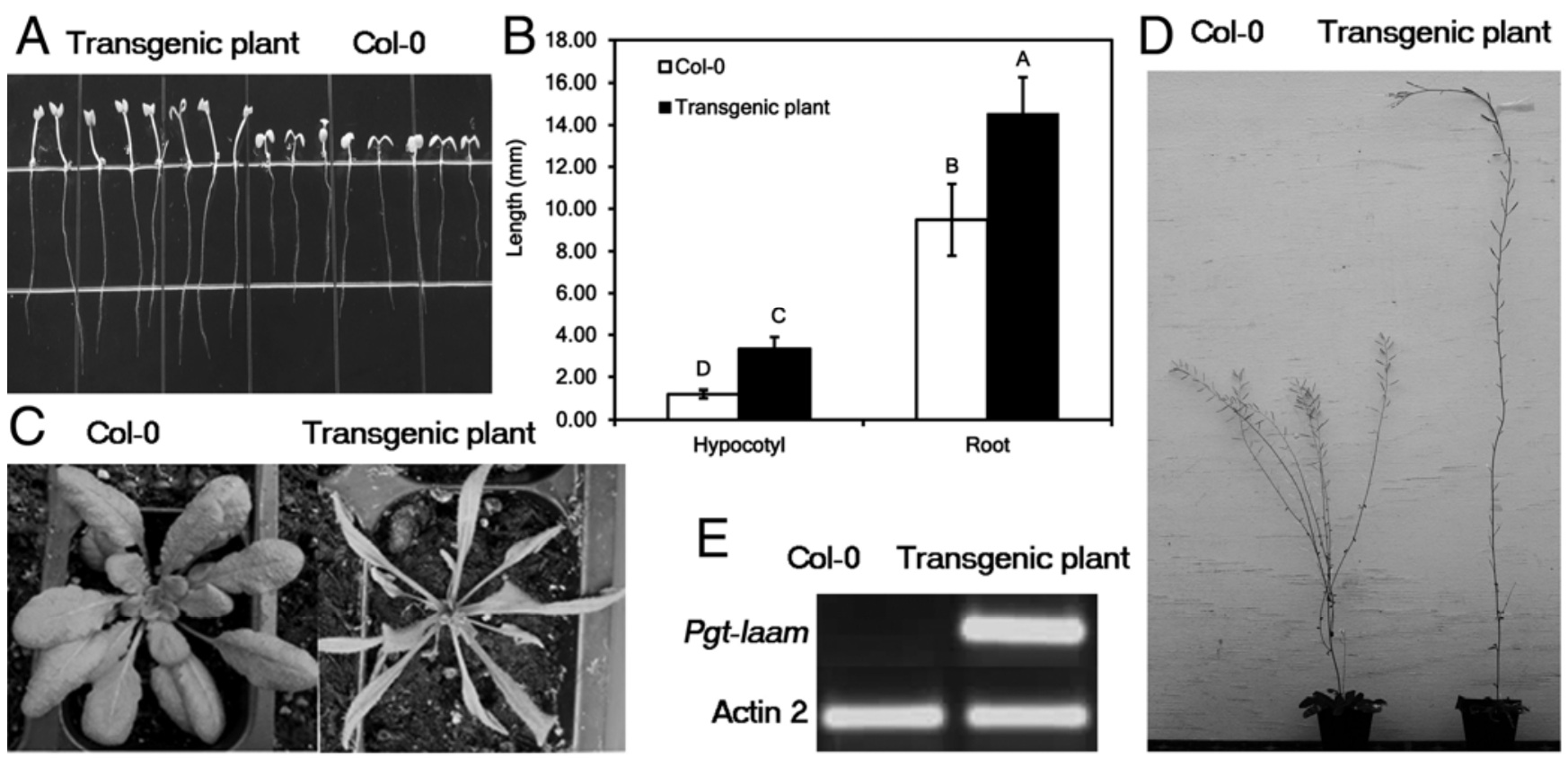

Fig. 4. Transgenic Arabidopsis plants expressing Pgt-IaaM display phenotypes associated with high-auxin content. A, Hypocotyl and roots of 5-day-old transgenic seedlings and wild type Col-0; B, 5-day-old transgenic seedlings display elongated hypocotyls and roots. White bars: wild type; shaded bars: transgenic plants. Values are means and standard deviations of three replicates $(n>50)$. Different letters above columns indicate significant differences $(P \leq$ $0.05 ; t$ test with $\alpha=0.05)$. C, Four-week-old transgenic plants display narrow and downward-curling leaves. D, Seven-week-old transgenic plants. E, reversetranscriptase polymerase chain reaction analysis of Pgt-IaaM gene expression in Arabidopsis. Col-0: wild type Arabidopsis; transgenic plant: transgenic Arabidopsis expressing Pgt-IaaM. Similar results were obtained for three independent transgenic lines. 


\section{DISCUSSION}

Proteins with haustoria-specific expression in rusts and other biotrophic fungi are considered likely candidates for important effectors because of the importance of these cells in acquiring nutrients and altering their host cell environment (Catanzariti et al. 2007; Dodds et al. 2009). Most of the attention on rust haustorial function to date has focused on proteins that function in nutrient uptake (Voegele et al. 2001) or small secreted proteins expressed in haustoria (HESPs) that potentially function in pathogenicity or interact with resistance proteins (Dodds et al. 2004; Catanzariti et al. 2006). Haustoria-specific expression of a gene encoding a protein involved in a hormone biosynthetic pathway indicates that Puccinia rust fungi make plant hormones as effectors. The Pgt-IaaM gene showed haustoria-specific expression. The experiments demonstrating that transient silencing of the gene reduced virulence indicates that production of IAM is an important component of pathogenicity in Puccinia graminis and possibly the other Puccinia spp. This virulence mechanism may not be utilized in all rust species because the IaaM homologs have not been identified in other rust genera, including $M$. larici-populina, whose genome has been sequenced (Duplessis et al. 2011).

The enzyme encoded by the IaaM genes makes IAM from tryptophan. The Pgt-IaaM gene enhanced IAM and IAA production when expressed in Arabidopsis and conferred a phenotype characteristic of auxin overproduction (Romano et al. 1993; Won et al. 2011; Zhao et al. 2001), indicating that the gene codes for a functional enzyme. A second enzyme, IAM hydrolase, encoded by an IaaH gene, is used by many microbes and possibly plants to make IAA. In plant-pathogenic bacteria and several fungi that make IAA via the IAM pathway, both genes are generally present in the genome (Patten and Glick 1996; Tsavkelova et al. 2012). The three Puccinia spp. for which genome sequence is now available are unusual in that only the gene encoding the first enzyme is present. This raises the question of how IAA production might be boosted with just the addition of one of the two enzymatic steps by the fungus. However, genes encoding proteins with IAM hydrolase activity have been identified in tobacco (NtAMII gene) and homologs are present in many species, including wheat (Mano et al. 2010). Alternative pathways may also be used to make IAA from IAM in Arabidopsis (Sugawara et al. 2009). In wheat, production of IAM may be rate limiting for IAA production by the IAM pathway or any other pathways that utilize IAM; however, this limitation is relieved in cells containing Puccinia rust haustoria.

Although most rust effector proteins are thought to be secreted from haustorial cells (Catanzariti et al. 2006, 2007; Kemen et al. 2005), it is not known where the IAA is actually synthesized in wheat cells harboring haustoria. The predicted protein sequences coded by the Pgt-IaaM gene had no apparent signal peptide to direct secretion of the protein. This sug- gests that IAM is made in the haustoria and, possibly, then enters the plant cell cytoplasm where the plant AMI1 protein is located (Pollmann et al. 2006) to make IAA. Small amounts of IAA were detected in $P$. graminis f. sp. tritici uredinial spores, indicating that $P$. graminis f. $\mathrm{sp}$. tritici can synthesize IAA. This is likely synthesized via another IAA biosynthetic pathway. Many fungi, including biotrophs and saprophytes, make IAA but multiple pathways are used. For example, $U$. maydis was found to make most of its IAA in culture by converting tryptophan to IAA via the intermediates indole-3-acetaldehyde and tryptamine (Reineke et al. 2008). P. graminis has genes homologous to $U$ maydis genes that function to catalyze both steps, although it is not known whether they function in IAA biosynthesis.

Plant pathogens enhance susceptibility to host plants by modulating the hormonal balance of the plant cells. However, relatively little is known about hormone involvement in the interactions between plants and cereal rust fungi. In the Puccinia-wheat system, small amounts of IAA were detected in plants at $2 \mathrm{dpi}$, and then free IAA levels noticeably increased by $4 \mathrm{dpi}$ as the infections spread through the plant tissues and the free IAA levels increased to approximately $5 \mathrm{ng} / \mathrm{g} \mathrm{FW}$ by 6 dpi. The low levels of IAA early in infection may be partly because relatively few haustoria have been established but it is also possible that the initial slight increase of local IAA concentration stimulates the host plants to further amplify the auxin biosynthesis pathway. The demonstration of the functionality of the Pgt-IaaM gene and haustoria-specific expression indicates that the fungus is modulating the increase of IAA in the host cells and a synergistic effect between $P$. graminis f. sp. tritici- and plant-derived auxin may exist. The observed increased bacterial susceptibility in Arabidopsis expressing Pgt-IaaM along with previous work showing susceptibility linked to auxin overproduction agrees with this interpretation. How localized increases in auxin make the plant more susceptible is open to question. Previous work showing that pretreating rice plants with IAA increased susceptibility to bacterial and fungal pathogens associated the treatment with loosening of the cell wall, the natural protective barrier of plant cells to invaders (Ding et al. 2008; Fu et al. 2011). It remains to be seen whether cell wall loosening occurs in plant cells associated with rust haustoria.

ABA levels were also increased in $P$. graminis f. sp. triticichallenged wheat tissues in a similar pattern to IAA. Alternatively, levels of the cytokinin $\mathrm{tZ}$ dropped sharply. Biosynthetic pathways for these compounds in plants are complicated; therefore, it is not clear whether these concentration changes are directly mediated by the rust pathogen or a plant response to infection. There is precedence for the levels of either type of hormone to affect resistance. ABA is generally associated in plant responses to abiotic stresses but effects on resistance have been noted. Pretreating plants with exogenous ABA increased plant susceptibility to bacterial and fungal pathogens

Table 1. Indole-3-acetic acid (IAA) and indole-3-acetamide (IAM) levels in different developmental stages of wild type Arabidopsis (Col-0) and transgenic plants $^{\mathrm{a}}$

\begin{tabular}{|c|c|c|c|}
\hline Plants & Organ tissues & Free IAA (pg/g FW) & IAM (ng/g FW) \\
\hline Col-0 & 10-day-old seedlings & $2.45 \pm 1.22$ & $0.00 \pm 0.00$ \\
\hline Transgenic plant & $\ldots$ & $15.53 \pm 5.79$ & $2.37 \pm 0.24$ \\
\hline Col-0 & 4-week-old rosette leaves & $8.85 \pm 7.11$ & $0.17 \pm 0.06$ \\
\hline Transgenic plant & $\ldots$ & $74.69 \pm 11.85$ & $3.36 \pm 0.73$ \\
\hline Col-0 & 6-week-old cauline leaves & $16.63 \pm 5.62$ & $0.42 \pm 0.17$ \\
\hline Transgenic plant & $\ldots$ & $54.96 \pm 16.94$ & $5.71 \pm 0.18$ \\
\hline Col-0 & Flowers & $130.28 \pm 39.09$ & $0.16 \pm 0.08$ \\
\hline Transgenic plant & $\ldots$ & $249.60 \pm 47.30$ & $5.72 \pm 0.57$ \\
\hline
\end{tabular}

${ }^{a}$ Specified organ tissues from Col-0 and transgenic plants were harvested and used for IAA and IAM measurement. Values are means and standard errors of three replicates of Col-0 and a single transgenic line. Three independent transgenic lines were investigated with similar results. 
(De Torres-Zabala et al. 2007; Mohr and Cahill 2003; Thaler and Bostock 2004). De Torres-Zabala and associates (2007) found that genes of Arabidopsis upregulated by effector delivery from DC3000 shared a $42 \%$ overlap with ABA-responsive genes, suggesting that one of the effector functions is to elevate components of the ABA signaling and biosynthesis pathways to promote virulence. Additionally, ABA-deficient tomato mutants had enhanced resistance to Botrytis cinerea (Audenaert et al. 2002). Work with bacterial and downy mildew pathogens of Arabidopsis have indicated that increases in cytokinin levels increase plant immunity (Argueso et al. 2012; Choi et al. 2010). The observed increases in ABA and decreases in cytokinin are consistent with the idea that these changes would benefit the pathogen and could conceivably be directly mediated by it. Further examination of the rust genomes may identify enzymes that affect the plant's metabolic pathways for these compounds.

BSMV-mediated HIGS has emerged as a powerful tool to study the functions and importance of candidate genes from biotrophic fungi because it enables knockdown of expression of target genes without stable transformation of the pathogen or host (Nowara et al. 2010; Panwar et al. 2013; Yin et al. 2011). Yin and associates (2011) showed that the approach could be used to silence most genes that were expressed mainly in haustoria. The results indicated that the silencing occurred mainly in $P$. striiformis haustorial cells as compared with other hyphal cells growing in the plant and that silencing of most genes did not affect fungal pathogenicity or disease development. Panwar and associates (2013) demonstrated that targeting essential genes in $P$. triticina could reduce the pathogenicity or development of the fungus and, thus, make the plant more resistant. The present study found that the HIGS phenomenon also works in $P$. graminis and identified a target gene, Pgt-IaaM, as essential to full pathogenicity. The IaaM genes in the three Puccinia spp. are highly conserved (approximately $80 \%$ identical in amino acids) and lie on partially syntenic chromosomal regions, indicating that they were present in their genomes before the three species diverged and that they represent an ancient strategy for pathogenicity. Blocking the action of one of the more conserved pathogenicity mechanisms in transgenic cereals may have great potential for engineering durable resistance to multiple rust diseases in cereals.

\section{MATERIALS AND METHODS}

Plants materials, fungal races, and growth conditions.

The plants used in this study included McNair 701 wheat, the 'Sr31/6*LMPG' wheat that carries the $S r 31$ gene, and $A$. thaliana Col-0. McNair 701 was used for stem rust $P$. graminis f. sp. tritici gene-silencing assays, Sr31/6*LMPG was used as a stem-rust-resistant control, and Col-0 was used as a source of wild-type Arabidopsis for transformation experiments. Wheat seedlings for gene-silencing assays were sown in pots containing potting mix and placed in growth chambers, as previously described (Yin et al. 2011). Arabidopsis seed were surface sterilized and cold-treated as described by Sandhu and associates (2012) and sown on $1 / 2 \times$ Murashige and Skoog (MS) medium (PhytoTechnology Laboratories Inc., Shawnee Mission, KS, U.S.A.) containing $0.8 \%$ (wt/vol) phytablend (Caisson Laboratories Inc., Rexburg, ID, U.S.A.) and 1.5\% (wt/vol) sucrose, with appropriate antibiotics (kanamycin at $30 \mu \mathrm{g} / \mathrm{ml}$ ). Germinating Arabidopsis seed were incubated in darkness at $4^{\circ} \mathrm{C}$ for 4 days, then transferred to incubators with constant white light $\left(30 \mu \mathrm{mol} / \mathrm{m}^{2} / \mathrm{s}\right.$ ) for 1 week at $25^{\circ} \mathrm{C}$. For observation of root phenotypes and etiolated hypocotyls, $1 \%$ (wt/vol) phytagel (Sigma-Aldrich, St. Louis) plates were used in a vertical position. Arabidopsis seedlings were then transplanted to pots containing potting mix and placed in growth chambers with white light $\left(200 \mu \mathrm{mol} / \mathrm{m}^{2} / \mathrm{s}\right)$ set at $21^{\circ} \mathrm{C}$ and 60 to $70 \%$ humidity. Approximately $100 \mathrm{mg}$ of tissue from different developmental stages (10-day-old seedlings, 4-week-old rosette leaves, 6-weekold cauline leaves, and flowers) of Col-0 and transgenic Arabidopsis plants were harvested and stored at $-80^{\circ} \mathrm{C}$ for further use. Urediniospores of $P$. graminis CRL 75-36-700-3, race SCCL (Pgt7A), were increased on McNair 701 as previously described (Yin et al. 2011). Wheat leaves in different rustinfected stages $(2,4$, and 6 days) and healthy plants were harvested for hormone measurement. Fresh spores were collected and used in inoculation experiments or stored at $-80^{\circ} \mathrm{C}$ for RNA or hormone extraction.

Construction of BSMV-derived vector, in vitro transcription of viral RNAs, virus and rust inoculations, and rust disease assays.

BSMV $\gamma:$ Pgt-IaaM was constructed and viral RNAs were synthesized in vitro, as previously described (Yin et al. 2011).
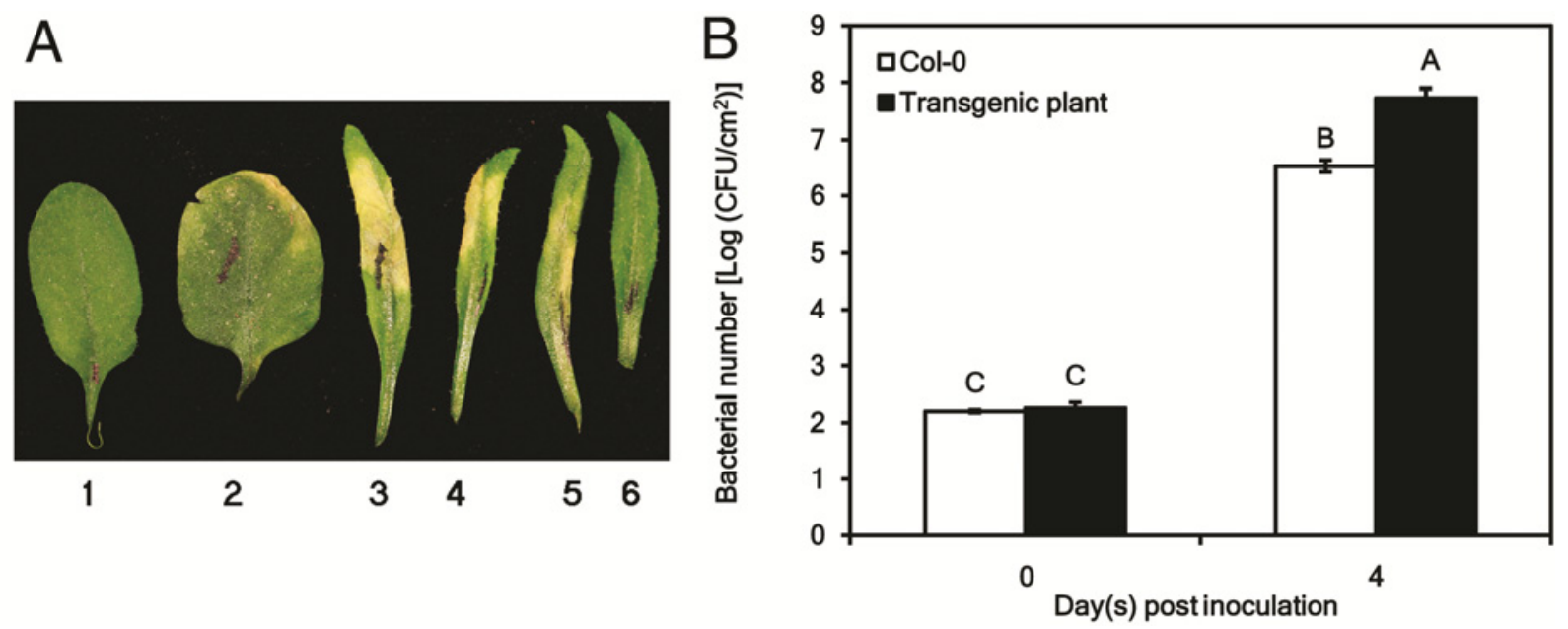

Fig. 5. Expression of Pgt-IaaM in Col-0 promotes susceptibility to Pseudomonas syringae DC3000. A, Disease symptoms of Pgt-IaaM transgenic plants and Col-0 at 4 days after inoculation with P. syringae pv. tomato DC3000. Lane 1, Col-0 mock inoculated; lane 2, Col-0 challenged with DC3000; lane 3, transgenic line 1 challenge with DC3000; lane 4, transgenic line 2 challenge with DC3000; lane 5, transgenic line 3 challenge with DC3000; lane 6, transgenic line 1 mock inoculated. B, Growth of DC3000 on transgenic plants and Col- 0 . White bars represent wild type and shaded bars represent transgenic plants. Different letters above columns indicate significant differences $(P \leq 0.05 ; t$ test with $\alpha=0.05)$. 
In brief, $179 \mathrm{bp}$ of coding sequence of Pgt-IaaM was amplified from cDNA of rust infected wheat leaves using primers $5^{\prime}$ ATAAGAATGCGGCCGCTAAACTATCAAGTCTTGGAGC ATTCACTCTGG-3' and 5'-CCTTAATTAAGGGACATTCA TGGAAGTCCTCAACGC-3'. The amplicons were double digested with NotI and PacI and directionally ligated into Not $\mathrm{I} / \mathrm{Pac} \mathrm{I}$ sites of the BSMV $\gamma$ vector. The derived $\mathrm{p} \gamma$ construct, $\mathrm{p} \alpha$, and $\mathrm{p} \beta \Delta \beta \mathrm{a}$ were linearized by BssHII, MluI, or SpeI digestion, respectively. In vitro transcripts were prepared from the three linearized plasmids using the mMessage mMachine $\mathrm{T} 7$ in vitro transcription kit (Ambion, Austin, TX, U.S.A.) following the manufacturer's instructions. First and second fully expand leaves of 12-day-old McNair 701 wheat plants were inoculated with the transcripts produced from the BSMV construct carrying the Pgt-IaaM gene fragment. By $10 \mathrm{dpi}$, when virus symptoms became apparent on newer uninoculated leaves, only those leaves displaying mild virus symptoms were inoculated with Pgt7A spores. The infected leaves were harvested for RNA extraction at 5 dpi. The other infected plants were kept in the growth chamber until $12 \mathrm{dpi}$, and the IT were assessed based on a 0-to-4 rating scale (Stakman et al. 1962). The BSMV:MCS construct was used as negative control. At least three independent experiments were conducted.

\section{RT-qPCR and RT-PCR analysis.}

RT-qPCR analysis was performed as described in Yin and associates $(2009,2011)$ to estimate $P$. graminis f. sp. tritici gene expression in different developmental stages (urediniospores, infected leaves, and purified haustoria) and also to estimate levels of gene expression after HIGS. To evaluate gene expression in different developmental stages, fresh urediniospores were collected from infected leaves at 12 to $14 \mathrm{dpi}$, infected leaves were harvested at $5 \mathrm{dpi}$, and haustoria were isolated from infected leaves at $5 \mathrm{dpi}$. Three biologically independent samples were used for each developmental stage. To evaluate the extent of gene silencing, the infected wheat leaves challenged with virus and rust were harvested at $5 \mathrm{dpi}$. Six biological replications were included for both the BSMV construct and the BSMV vector control constructs. The ratios of expression of each putative silenced seedling leaf were compared with each of the BSMV control seedlings, typically giving six estimates of silencing for each seedling. RT-qPCR was performed using $P$. graminis $\mathrm{f}$. sp. tritici-actin transcript to normalize the amount of cDNA in each of the samples, which were amplified with the primers $5^{\prime}$-TGTCGGGTGGAACGA CCATGTATT-3' and 5'-AGCCAAGATAGAACCACCGATC CA-3'.

RT-PCR was conducted to measure target gene expression levels in Arabidopsis transgenic lines. Total RNA was isolated from 4-week-old plant leaves. The actin 2 gene (At3g18780) was used as an internal control to normalize the amount of cDNA in each of the samples, which were amplified using the primers 5'-GACCTTTAACTCTCCCGCTATG-3' and 5'-GAG ACACACCATCACCAGAAT-3' in amplification reactions for 22 cycles. The Pgt-IaaM gene was amplified for 30 cycles using primers 5'-GGGCAACAAGAATGGGAAGA-3' and 5'-CCA CTAAGCGGCAGATGTAAG-3'. All reactions were repeated three times with consistent results.

\section{Expression of Pgt-IaaM in Arabidopsis.}

To generate the Pgt-IaaM expression construct, the coding region of Pgt-IaaM was amplified from cDNA of rust infected wheat leaves by PCR using primers 5'-GCGTCGACATGAAC TCCGTCAACTACCAAG-3' and 5'-AACTGCAG CATACAG TCATCTTTGAACACCAC-3'. The PCR product (1,790 bp) was digested with SalI and PstI and ligated into binary vector pCHF3 with the same restriction enzymes as previously de- scribed (Neff et al. 1999). The derived construct was electroporated into Agrobacterium tumefaciens GV3101. The A. tumefaciens strain carrying Pgt-IaaM was transformed into Col-0 according to the floral dip method (Clough and Bent 1998). Multiple transformants were identified by screening on plates containing kanamycin at $30 \mu \mathrm{g} / \mathrm{ml}$. Three representative lines with high levels of Pgt-IaaM expression (based on RT-PCR analysis described above) were chosen for further analysis.

\section{Roots and hypocotyl length of Arabidopsis measurements.}

To measure hypocotyl length, 5-day-old seedlings grown on $1 / 2$ MS media with $1 \%(\mathrm{wt} / \mathrm{vol})$ phytagel were removed from plates and placed on transparent sheets. The seedlings were digitized with a flatbed scanner at a resolution of $600 \mathrm{dpi}$. The hypocotyls and root were measured from the scanned images that included a 1-mm scaled ruler and Image J 1.29J (National Institutes of Health, Bethesda, MD, U.S.A.). All experiments were done in triplicate $(n \geq 50)$.

\section{Hormone measurements.}

The plant materials (described above) and urediniospores (approximately $100 \mathrm{mg} \mathrm{FW}$ ) were placed in 1.7- $\mathrm{ml}$ microcentrifuge tubes and extracted in $1.0 \mathrm{ml}$ of Bieleski solvent (methanol/chloroform/formic acid/water, 12:5:2:1) using a TissueLyser II (Qiagen, Valencia, CA, U.S.A.) at a frequency of $27 \mathrm{~Hz}$ for $3 \mathrm{~min}$ after adding two 2.8-mm-diameter steel balls. The tube content was ultrasonicated for $3 \mathrm{~min}$ and then stirred for $10 \mathrm{~min}$ at $4^{\circ} \mathrm{C}$. After centrifugation (10 min, 15,000 $\mathrm{rpm}, 4^{\circ} \mathrm{C}$ ), the supernatants were lyophilized. The dried extracts were dissolved in $50 \mu \mathrm{l}$ of mobile phase (acetonitrile/ water [5:95], $0.1 \%$ formic acid) prior to ultra-high-performance liquid chromatography (UPLC) tandem mass spectrometry (MS/MS) analyses. Plant hormones were measured with a UPLC electrospray ionization quantitative MS/MS (AQUITY UPLC System/XEVO TQ; Waters, Milford, MA, U.S.A.) with an HSS column (AQUITY UPLC HSS T3, $1.8 \mu \mathrm{m}, 2.1$ by 100 $\mathrm{mm}$; Waters). Plant hormones were separated at a flow rate of $0.3 \mathrm{ml} \mathrm{min} \mathrm{m}^{-1}$ with linear gradients of solvent A $(0.1 \%$ formic acid) and solvent $\mathrm{B}$ ( $0.1 \%$ formic acid in acetonitrile) set according to the following profile: $0 \mathrm{~min}, 95 \% \mathrm{~A} ; 0.5 \mathrm{~min}$, $95 \% \mathrm{~A} ; 7.0 \mathrm{~min}, 50 \% \mathrm{~A} ; 7.5 \mathrm{~min}, 5 \% \mathrm{~A} ; 10 \mathrm{~min}, 5 \% \mathrm{~A} ; 10.5$ $\min , 95 \% \mathrm{~A}$; and $13 \mathrm{~min}, 95 \% \mathrm{~A}$. Capillary voltage was 2.5 $\mathrm{kV}$. Selective multiple reaction monitoring mode using massto-charge $(\mathrm{m} / \mathrm{z})$ transitions of precursor and product ions was performed $(\mathrm{m} / \mathrm{z} 176.1 \rightarrow 130.0$ for IAA, $175.1 \rightarrow 103.1$ for IAM, $265.2 \rightarrow 135.0$ for ABA, and $220.1 \rightarrow 136.1$ for $t Z$ ). Cone voltage $(\mathrm{V})$ and collision energy $(\mathrm{eV})$ were as follows: IAA: $18 \mathrm{~V}, 24 \mathrm{eV}$; IAM: $18 \mathrm{~V}, 26 \mathrm{eV}$; ABA: $28 \mathrm{~V}, 12 \mathrm{eV}$; and tZ: $34 \mathrm{~V}$, $20 \mathrm{eV}$. Data were processed by MassLynx software with TargetLynx (version 4.1; Waters). Stable isotope-labeled standard compounds were purchased from OlChemim Ltd. (Olomouc, Czech Republic) and standard compounds were purchased from Sigma-Aldrich.

\section{Bacterial growth and disease assays on transgenic Arabidopsis.}

Pseudomonas syringae pv. tomato DC3000 was grown on King's B medium containing rifampicin $(50 \mu \mathrm{g} / \mathrm{ml})$ and ampicillin $(50 \mu \mathrm{g} / \mathrm{ml})$ overnight at $28^{\circ} \mathrm{C}$. Cultures were centrifuged at $5,000 \times g$ for $10 \mathrm{~min}$, and bacterial pellets were washed twice with sterile double-distilled $\mathrm{H}_{2} \mathrm{O}$ and resuspended in 10 $\mathrm{mM} \mathrm{MgCl}$ for plant inoculations. The bacterial disease assays were performed as previously described (Xiao et al. 2007). In brief, leaves of 4-week-old Arabidopsis thaliana transgenic plants and wild-type Col-0 were infiltrated with $P$. syringae DC3000 at $10^{4} \mathrm{CFU} / \mathrm{ml}$ using a needleless syringe, and bacterial growth was monitored at 0 and 4 dpi using serial dilution 
plating of ground leaf disks. Six leaf discs $\left(0.5 \mathrm{~cm}^{2}\right.$ each $)$ from three inoculated plants were collected with a cork borer. Two discs were ground in $1 \mathrm{ml}$ of sterile water, diluted to the desired concentration, and plated on tryptic soy agar medium containing rifampicin at $50 \mu \mathrm{g} / \mathrm{ml}$ and ampicillin at $50 \mu \mathrm{g} / \mathrm{ml}$, then cultured at $28^{\circ} \mathrm{C}$ for $48 \mathrm{~h}$, and cell numbers were counted. Disease symptoms on Arabidopsis leaves were photographed 4 dpi. Three independent experiments were conducted.

\section{Statistical analysis.}

Data analyses were performed using the general linear model procedures on SAS statistical software (SAS Institute, Inc., Cary, NC, U.S.A.). Multiple comparisons were performed by the Tukey's test or $t$ test $(P \leq 0.05)$. Significance was accepted at $\alpha=0.05$.

\section{ACKNOWLEDGMENTS}

This work was supported by National Institute of Food and Agriculture award number 2012-67013-19400 and the Washington Grain Commission. We thank M. Neff for providing Arabidopsis thaliana Col-0 seed and the binary vector pCHF3, K. Evans for assistance with photography, and X. Chen for assistance with rust culturing and facilities.

\section{LITERATURE CITED}

Argueso, C. T., Ferreira, F. J., Epple, P., To, J. P., Hutchison, C. E., Schaller, G. E., Dangl, J. L., and Kieber, J. J. 2012. Two-component elements mediate interactions between cytokinin and salicylic acid in plant immunity. PLoS Genet. 8:e1002448.

Artemenko, E. N., Umnov, A. M., and Chkanikov, D. I. 1980. Changes in the level of indolyl-3-acetic acid and possible paths of its regulation in leaves of wheat infected with stem rust. Sov. Plant Physiol. 81:121-126. (English translation)

Audenaert, K., De Meyer, G. B., and Hofte, M. M. 2002. Abscisic acid determines basal susceptibility of tomato to Botrytis cinerea and suppresses salicylic acid-dependent signaling mechanisms. Plant Physiol. 128:491-501.

Bari, R., and Jones, J. D. G. 2009. Role of plant hormones in plant defence responses. Plant Mol. Biol. 69:473-488.

Birch, P. R., Armstrong, M., Bos, J., Boevink, P., Gilroy, E. M., Taylor, R. M., Wawra, S., Pritchard, L., Conti, L., Ewan, R., Whisson, S. C., van West, P., Sadanandom, A., Kamoun, S. 2009. Towards understanding the virulence functions of RXLR effectors of the oomycete plant pathogen Phytophthora infestans. J. Exp. Bot. 60:1133-1140.

Bushnell, W. R. 1984. Structural and physiological alterations in susceptible host tissue. Pages 477-507 in: The Cereal Rusts: Volume 1. Origins, Specificity, Structure, and Physiology. W. R. Bushnell and A. P. Roelfs, eds. Academic Press Inc., Orlando, FL, U.S.A.

Catanzariti, A. M., Dodds, P. N., Lawrence, G. J., Ayliffe, M. A., and Ellis, J. G. 2006. Haustorially-expressed secreted proteins from flax rust are highly enriched for avirulence elicitors. Plant Cell 18:243-256.

Catanzariti, A.-M., Dodds, P. N., and Ellis, J. G. 2007. Avirulence proteins from haustoria-forming pathogens. FEMS (Fed. Eur. Microbiol. Soc.) Microbiol. Lett. 269:181-188.

Chen, Z. Kloek, A. P., Cuzick, A., Moeder, W., Tang, D., Innes, R. W., Klessig, D. F., McDowell, J. M., and Kunkel, B. N. 2004. The Pseudomonas syringae type III effector AvrRpt2 functions downstream or independently of SA to promote virulence on Arabidopsis thaliana. Plant J. 37:494-504.

Chen, Z., Agnew, J. L., Cohen, J. D., He, P., Shan, L., Sheen, J., and Kunkel, B. N. 2007. Pseudomonas syringae type III effector AvrRpt2 alters Arabidopsis thaliana auxin physiology. Proc. Natl. Acad. Sci. U.S.A. 104:20131-20136.

Choi, J., Huh, S. U., Kojima, M., Sakakibara, H., Paek, K. H., and Hwang, I. 2010. The cytokinin-activated transcription factor ARR2 promotes plant immunity via TGA3/NPR1-dependent salicylic acid signaling in Arabidopsis. Dev. Cell. 19:284-295.

Clark, E., Manulis, S., Ophir, Y., Barash, I., and Gafni, Y. 1993. Cloning and characterization of iaaM and iaaH from Erwinia herbicola pathovar gypsophilae. Phytopathology 83:234-240.

Clough, S. J., and Bent, A. F. 1998. Floral dip: A simplified method for Agrobacterium-mediated transformation of Arabidopsis thaliana. Plant J. 16:735-743.

Comai, L., and Kosuge, T. 1982. Cloning characterization of iaaM, a viru- lence determinant of Pseudomonas savastanoi. J. Bacteriol. 149:40-46. Cui, F., Wu, S., Sun, W., Coaker, G., Kunkel, B., He, P., and Shan, L. 2013. Pseudomonas syringae type III effector AvrRpt2 promotes pathogen virulence via stimulating Arabidopsis Aux/IAA protein turnover. Plant Physiol. 162:1018-1029.

De Torres-Zabala, M., Truman, W., Bennett, M. H., Lafforgue, G., Mansfield, J. W., Rodriguez Egea, P., Bögre, L., and Grant, M. 2007. Pseudomonas syringae pv. tomato hijacks the Arabidopsis abscisic acid signaling pathway to cause disease. EMBO (Eur. Mol. Biol. Organ.) J. 26:1434-1443.

De Wit, P. J., Mehrabi, R., Van den Burg, H. A., and Stergiopoulos, I. 2009. Fungal effector proteins: Past, present and future. Mol. Plant Pathol. 10:735-747.

Ding, X., Cao, Y., Huang, L., Zhao, J., Xu, C., Li, X., and Wang, S. 2008. Activation of the indole-3-acetic acid-amido synthetase GH3-8 suppresses expansin expression and promotes salicylate- and jasmonateindependent basal immunity in rice. Plant Cell 20:228-240.

Dodds, P. N., Lawrence, G. J., Catanzariti, A. M., Ayliffe, M.A., and Ellis, J. G. 2004. The Melampsora lini AvrL567 avirulence genes are expressed in haustoria and their products are recognized inside plant cells Plant Cell 16:755-768.

Dodds, P. N., Rafiqi, M., Gan, P. H. P., Hardham, A. R., Jones, D. A., and Ellis, J. G. 2009. Effectors of biotrophic fungi and oomycetes: Pathogenicity factors and triggers of host resistance. New Phytol. 183:9931000.

Duplessis, S., Cuomo, C. A, Lin, Y.-C., Aerts, A., Tisserant, E., VeneaultFourrey, C., Joly, D. L., Hacquard, S., Amselem, J., Cantarel, B. L., Chiu, R., Coutinho, P. M., Feau, N., Field, M., Frey, P., Gelhaye, E., Goldberg, J., Grabherr, M. G., Kodira, C. D., Kohler, A., Kües, U., Lindquist, E. A., Lucas, S. M., Mago, R., Mauceli, E., Morin, E., Murat, C., Pangilinan, J. L., Park, R., Pearson, M., Quesneville, H., Rouhier, N., Sakthikumar, S., Salamov, A. A., Schmutz, J., Selles, B., Shapiro, H., Tanguay, P., Tuskan, G. A., Henrissat, B., Van de Peer, Y., Rouzé, P., Ellis, J. G., Dodds, P. N., Schein, J. E., Zhong, S., Hamelin, R. C., Grigoriev, I. V., Szabo, L. J., and Martin, F. 2011. Obligate biotrophy features unraveled by the genomic analysis of rust fungi. Proc. Natl. Acad. Sci. U.S.A. 108:9166-9171.

Fu, J., Liu, H., Li, Y., Yu, H., Li, X., Xiao, J., and Wang, S. 2011. Manipulating broad-spectrum disease resistance by suppressing pathogeninduced auxin accumulation in rice. Plant Physiol. 155:589-602.

Glazebrook, J. 2005. Contrasting mechanisms of defense against biotrophic and necrotrophic pathogens. Annu. Rev. Phytopathol. 43:205227.

Grant, M., and Lamb, C. 2006. Systemic immunity. Curr. Opin. Plant Biol 9:414-420.

Kazan, K., and Manners, J. M. 2009. Linking development to defense: Auxin in plant-pathogen interactions. Trends Plant Sci. 14:373-382.

Kemen, E., Keman, A. C., Rafiqi, M., Hempel, U., Mendgen, K., Hahn, M., and Voegele, R. T. 2005. Identification of a protein from rust fungi transferred from haustoria into infected plant cells. Mol. Plant-Microbe Interact. 18:1130-1139.

Koeck, M., Hardham, A. R., and Dodds, P. N. 2011. The role of effectors of biotrophic and hemibiotrophic fungi in infection. Cell Microbiol. 13:1849-1857.

Mano, Y., Nemoto, K., Suzuki, M., Seki, H., Fujii, I., and Muranaka, T., 2010. The AMI1 gene family: Indole-3-acetamide hydrolase functions in auxin biosynthesis in plants. J. Exp. Bot. 61:25-32.

Manulis, S., Valinski, L., Gafni, Y., and Hershenhorn, J. 1991. Indole-3acetic acid biosynthetic pathways in Erwinia herbicola in relation to pathogenicity on Gypsophila paniculata. Physiol. Mol. Plant Pathol. 39:161-171.

Maor, R., Haskin, S., Kedmi-Levi, H., and Sharon, A., 2004. Biosynthesis, regulation and in planta auxin production by Colletotrichum gloeosporioides f. sp. aeschynomene. Appl. Environ. Microbiol. 69:16951701.

Mohr, P., and Cahill, D. 2003. Abscisic acid influences the susceptibility of Arabidopsis thaliana to Pseudomonas syringae pv. tomato and Peronospora parasitica. Funct. Plant Biol. 30:461-469.

Morgan, W., and Kamoun, S. 2007. RXLR effectors of plant pathogenic oomycetes. Curr. Opin. Microbiol. 10:332-338.

Mutka, A. M., Fawley, S., Tsao, T., and Kunkel, B. N. 2013. Auxin promotes susceptibility to Pseudomonas syringae via a mechanism independent of suppression of salicylic acid-mediated defenses. Plant J. 74:746-54.

Nakamura, T., Tomita, K., Kawanabe, Y., and Murayama, T. 1982. Effect of auxin and gibberellin on spore germination in Neurospora crassa II. "Spore density effect" and auxin. Plant Cell Physiol. 23:1363-1369.

Navarro, L., Dunoyer, P., Jay, F., Arnold, B., Dharmasiri, N., Estelle, M. Voinnet, O., and Jones J. D. G. 2006. Plant miRNA contributes to antibacterial resistance by repressing auxin signaling. Science 312:436-439. 
Neff, M. M., Nguyen, S. M., Malancharuvil, E. J., Fujioka, S., Noguchi, T., Seto, H., Tsubuki, M., Honda, T., Takatsuto, S., Yoshida, S., and Chory, J. 1999. BAS1: A gene regulating brassinosteroid levels and light responsiveness in Arabidopsis. Proc. Natl. Acad. Sci. U.S.A. 96:15316-15323.

Nirmala, J., Drader, T., Lawrence, P. K., Yin, C., Hulbert, S., Steber, C. M., Steffenson, B. J., Szabo, L. J., von Wettstein, D., and Kleinhofs, A. 2011. Concerted action of two avirulent spore effectors activates reaction to Puccinia graminis ( $\mathrm{Rpg} 1)$-mediated cereal stem rust resistance. Proc. Natl. Acad. Sci. U.S.A. 108:14676-14681.

Nowara, D., Gay, A., Lacomme, C., Shaw, J., Ridout, C., Douchkov, D., Hensel, G., Kumlehn, J., and Schweizer, P. 2010. HIGS: Host-induced gene silencing in the obligate biotrophic fungal pathogen Blumeria graminis. Plant Cell 22:3130-3141.

Panwar, V., McCallum, B., and Bakkeren, G. 2013. Host-induced gene silencing of wheat leaf rust fungus Puccinia triticina pathogenicity genes mediated by the Barley stripe mosaic virus. Plant Mol. Biol. 81:595608

Patten, C., and Glick, B. 1996. Bacterial biosynthesis of indole-3-acetic acid. Can. J. Microbiol. 42:207-220.

Pollmann, S., Neu, D., Lehmann, T., Berkowitz, O., Schafer, T., and Weiler, E. W. 2006. Subcellular localization and tissue specific expression of amidase 1 from Arabidopsis thaliana. Planta 224:1241-1253.

Prusty, R., Grisafi, P., and Fink, G. R. 2004. The plant hormone indoleacetic acid induces invasive growth in Saccharomyces cerevisiae. Proc. Natl. Acad. Sci. U.S.A. 101:4153-4157.

Rao, R. P., Hunter, A., Kashpur, O., and Normanly, J. 2010. Aberrant synthesis of indole-3-acetic acid in Saccharomyces cerevisiae triggers morphogenic transition, a virulence trait of pathogenic fungi. Genetics 185:211-220

Reineke, G., Heinze, B., Schirawski, J., Buettner, H., Kahmann, R., and Basse, C. W. 2008. Indole-3-acetic acid (IAA) biosynthesis in the smut fungus Ustilago maydis and its relevance for increased IAA levels in infected tissue and host tumour formation. Mol. Plant Pathol. 9:339355.

Ridout, C. J., Skamnioti, P., Porritt, O., Sacristan, S., Jones, J. D., and Brown, J. K. 2006. Multiple avirulence paralogues in cereal powdery mildew fungi may contribute to parasite fitness and defeat of plant resistance. Plant Cell 18:2402-2414.

Robert-Seilaniantz, A., Grant, M., and Jones, J. D. 2011. Hormone crosstalk in plant disease and defense: More than just jasmonate-salicylate antagonism. Annu. Rev. Phytopathol. 49:317-343.

Robinson, M., Riov, J., and Sharon, A., 1998. Indole-3-acetic acid biosynthesis in Colletotrichum gloeosporioides f. sp. aeschynomene. Appl. Environ. Microbiol. 64:5030-5032.

Romano, C. P., Cooper, M. L., and Klee, H. J. 1993. Uncoupling auxin and ethylene effects in transgenic tobacco and Arabidopsis plants. Plant Cell 5:181-189.

Sandhu, K. S., Hagely, K., and Neff, M. M. 2012. Genetic interactions between brassinosteroid-inactivating $\mathrm{P} 450$ s and photomorphogenic photoreceptors in Arabidopsis thaliana. G3 (Bethesda) 2:1585-1593.

Schmidt, S. M., and Panstruga, R. 2011. Pathogenomics of fungal plant parasites: What have we learnt about pathogenesis? Curr. Opin. Plant Biol. 14:392-399.

Smidt, M., and Kosuge, T. 1978. The role of indole-3-acetic acid accumu- lation by alpha methyl tryptophan resistant mutants of Pseudomonas savastonoi in gall formation on oleanders. Physiol. Plant Pathol. 13:203-214.

Spaepen, S., and Vanderleyden J. 2011. Auxin and plant-microbe interactions. Cold Spring Harb. Perspect. Biol. 3. doi:pii:a001438. Published online.

Stakman, E. C., Stewart, D. M., and Loegering, W. Q. 1962. Identification of physiologic races of Puccinia graminis var. tritici. Agric. Res. Serv. E 617. U. S. Dep. Agric. Washington, DC.

Sugawara, S., Hishiyama, S., Jikumaru, Y., Hanada, A., Nishimura, T., Koshiba, T., Zhao, Y., Kamiya, Y., and Kasahara, H. 2009. Biochemical analyses of indole-3-acetaldoxime-dependent auxin biosynthesis in Arabidopsis. Proc. Nat. Acad. Sci. U.S.A. 106:5430-5435.

Surico, G., Iacobellis, N. S., and Sisto, A. 1985. Studies on the role of indole-3-acetic acid and cytokinins in the formation of knots on olive and oleander plants by Pseudomonas syringae pv. savastanoi. Physiol. Plant Pathol. 26:309-320.

Thaler, J. S., and Bostock, R. M. 2004. Interactions between abscisicacidmediated responses and plant resistance to pathogens and insects. Ecology 85:48-58.

Tsavkelova, E., Oeser, B., Oren-Young, L., Israeli, M., Sasson, Y., Tudzynski, B., and Sharon, A. 2012. Identification and functional characterization of indole-3-acetamide-mediated IAA biosynthesis in plantassociated Fusarium species. Fungal Genet. Biol. 49:48-57.

Voegele, R. T., Struck, C., Hahn, M., and Mendgen, K. 2001. The role of haustoria in sugar supply during infection of broad bean by the rust fungus Uromyces fabae. Proc. Natl. Acad. Sci. U.S.A. 98:8133-8138.

Wang, D., Pajerowska-Mukhtar, K., Culler, A. H., and Dong, X. 2007. Salicylic acid inhibits pathogen growth in plants through repression of the auxin signaling pathway. Curr. Biol. 17:1784-1790.

Won, C., Shen, X., Mashiguchi, K., Zheng, Z., Dai, X., Cheng, Y., Kasahara, H., Kamiya, Y., Chory, J., and Zhao, Y. 2011. Conversion of tryptophan to indole-3-acetic acid by TRYPTOPHAN AMINOTRANSFERASES OF ARABIDOPSIS and YUCCAs in Arabidopsis. Proc. Natl. Acad. Sci. U.S.A. 108:18518-18523.

Xiao, Y., Lan, L., Yin, C., Deng, X., Baker, D., Zhou, J. M., and Tang, X. 2007. Two-component sensor RhpS promotes induction of Pseudomonas syringae type III secretion system by repressing negative regulator RhpR. Mol. Plant-Microbe Interact. 20:223-234.

Yin, C., Chen, X., Wang, X., Han Q. M., Kang, Z., and Hulbert, S. H. 2009. Generation and analysis of expression sequence tags from haustoria of the wheat stripe rust fungus Puccinia striiformis f. sp. tritici. BMC Genomics 10:626.

Yin, C., Jurgenson, J. E., and Hulbert, S. H. 2011. Development of a hostinduced RNAi system in the wheat stripe rust fungus Puccinia striiformis f. sp. tritici. Mol. Plant-Microbe Interact. 24:554-561.

Zhao, Y., Christensen, S. K., Fankhauser, C., Cashman, J. R., Cohen, J. D. Weigel, D., and Chory, J. 2001. A role for flavin monooxygenase-like enzymes in auxin biosynthesis. Science 291:306-309.

\section{AUTHOR-RECOMMENDED INTERNET RESOURCES}

Broad Institute website: www.broadinstitute.org

National Institutes of Health Image $\mathbf{J}$ software website: rsb.info.nih.gov/ij 